\title{
FEATURES OF EXCITATION AND AZIMUTHAL AND MERIDIONAL PROPAGATION OF LONG-PERIOD Pi3 OSCILLATIONS OF THE GEOMAGNETIC FIELD ON DECEMBER 8, 2017
}

\author{
A.V. Moiseev \\ Yu. G. Shafer Institute of Cosmophysical Research \\ and Aeronomy SB RAS, \\ Yakutsk,Russia,moiseev@ikfia.ysn.ru \\ S.A. Starodubtsev \\ Yu. G. Shafer Institute of Cosmophysical Research \\ and Aeronomy SB RAS, \\ Yakutsk,Russia,starodub@ikfia.ysn.ru
}

\author{
V.V. Mishin \\ Institute of Solar-Terrestrial Physics SB RAS, \\ Irkutsk,Russia,vladm@iszf.irk.ru
}

\begin{abstract}
We study the Pi3 pulsations (with a period $T=15-30 \mathrm{~min}$ ) that were recorded on December 8, 2017 at ground stations in the midnight sector of the magnetosphere at the latitude range of DP2 current system convective electrojets. We have found that Pi3 are especially pronounced in the pre-midnight sector with amplitude of up to $300 \mathrm{nT}$ and duration of up to $2.5 \mathrm{hrs}$. The pulsation amplitude rapidly decreased with decreasing latitude from $\Phi^{\prime}=72^{\circ}$ to $\Phi^{\prime}=63^{\circ}$. The event was recorded during the steady magnetospheric convection. In the southward $B_{z}$ component of the interplanetary magnetic field, irregular oscillations were detected in the Pi3 frequency range. They correspond to slow magnetosonic waves occurring without noticeable variations in the dynamic pressure $P_{\mathrm{d}}$. Groundbased geomagnetic observations have shown azimuthal propagation of pulsations with a $0.6-10.6 \mathrm{~km} / \mathrm{s}$ velocity eastward and westward of the midnight meridian. An analysis of the dynamics of pulsations along the meridian has revealed their propagation to the equator at a velocity 0.75
\end{abstract}

\section{INTRODUCTION}

It is known that a magnetospheric substorm can generate geomagnetic pulsations in the range from 0.5 to $100 \mathrm{mHz}$ whose low-frequency part consists of Pi3 geomagnetic pulsations - irregular long-period $(T=600 \div 900 \mathrm{~s})$ geomagnetic field oscillations [Saito, Matsushita, 1967]. Our interest in these pulsations is due to the fact that it is still largely inexplicable phenomenon, and, furthermore, they have the greatest power spectral density of geomagnetic oscillations during substorms. This means that they are the main energy transporters between the ionosphere and the magnetosphere. Therefore, we can assume that Pi3 pulsations play the most important role in exciting the high-latitude ionosphere, causing ions to move, plasma irregularities to form, etc. Thus, examining properties of these pulsations is of great interest in researching the evolution of substorm phenomena in the geomagnetic field.

A number of studies have attributed the origin of $\mathrm{Pi} 3$ pulsation to the processes occurring in Earth's inner magnetosphere. Particularly important among these processes are magnetic field line resonances during the substorm growth phase [Keiling, 2009], various mechanisms associated with the magnetosphere-ionosphere
$7.87 \mathrm{~km} / \mathrm{s}$. In the projection onto the magnetosphere, the velocities are close in magnitude to the observed propagation velocities of substorm injected electrons. In the dawnside magnetosphere during ground-observed Pi3 pulsations, compression mode oscillations were recorded.

We conclude that propagation of geomagnetic field oscillations in this event depends on the dynamics of particle injections under the action of a large-scale electric field of magnetospheric convection, which causes earthward plasma movement due to reconnection in the magnetotail. Small-scale oscillations in the magnetosphere were secondary, excited by the solar wind oscillations penetrated into the magnetosphere.

Keywords: Pi3 pulsations, steady magnetospheric convection, convection electrojets, particle injections, azimuthal and meridional propagation, wave disturbances in the interplanetary medium.

coupling [Russell et al., 2013], reconnection in the magnetotail [Angelopoulos et al., 2002], as well as instabilities in the hot component of magnetospheric plasma [Mager et al., 2013], and currents associated with drift of particles injected during substorms [Guglielmi, Zolotukhina, 1980; Mager, Klimushkin, 2007].

At the same time, a relationship has been found between Pi3 pulsation and variations of interplanetary medium parameters [Moiseev et al., 2016; Parkhomov et al., 2018]. Alimaganbetov and Streltsov [2018] have conducted a statistical analysis of wave disturbances in the solar wind (SW) during substorms. They have analyzed 75 intense substorms and found out that wave disturbances with $0.6-0.7 \mathrm{mHz}$ frequencies often occur during substorms simultaneously in the magnetosphere and on Earth.

Study of wave disturbance propagation velocities and direction allows us to identify both their external (SW) and internal (magnetospheric) sources and transformation of different modes of oscillations during their generation.

Wygant et al. [2002] and Keiling et al. [2005] emphasize the role of MHD waves in energy transfer between the magnetotail and auroral zone, derived from observations at the plasma sheet boundary based on 
POLAR, CLUSTER, and FAST satellite measurements. These observations indicate that the Poynting vector flux, transferred by an Alfvén wave along the magnetic field lines at auroral latitudes, may be a dominant energy source during a substorm.

Efficiency of energy transfer by magnetosonic waves to the magnetosphere across its boundary has been studied by Leonovich et al. [2003]; the authors show that at flanks where the flow velocity is supersonic, the efficiency is $\geq 40 \%$, which far exceeds that on the dayside where the transfer coefficient is only 1-2\% [McKenzie, 1970]. Such efficient transfer of the Poynting vector flux can provide energetic SW - magnetosphere interaction not only under quiet conditions but also during moderate substorms. Thus, Mishin [1996], by an example of an isolated substorm at the latitude of Irkutsk, has demonstrated that the 8-min strong magnetosonic SW oscillations observed before the substorm could easily supply its power.

In this paper, we report results of the study of generation and propagation of $\mathrm{Pi} 3$ pulsations under steady magnetospheric convection in the December 8, 2017 event. This event was recorded under relatively quiet geomagnetic conditions, which allows us to examine in greater detail the direction and velocity of propagation of the geomagnetic pulsations.

\section{OBSERVATIONAL DATA}

To study characteristics of the geomagnetic pulsations, we have used geomagnetic observations from the well-known database SUPERMAG [Gjerloev, 2012] [http://supermag.jhuapl.edu/mag], as well as from projects MAGDAS [http://www.serc.kyushu-u.ac.jp/magdas] and THEMIS [http://themis.ssl.berkeley.edu/gmag_desc. shtml]. We have taken ACE, WIND, and THEMIS satellite measurements from the CDAWEB database [http://cdaweb.gsfc.nasa.gov]; and GOES observations, from the NOAA database [https://www.ngdc.noaa.gov/ stp/satellite/goes/dataaccess.html]. Coordinates of the satellites are listed in Table 1. Orbital elements of the satellites in the magnetosphere in the GSM coordinate system are shown in Figure 1. To study long-period variations of the magnetic field and plasma parameters, we have used 1-min averaged observational data. Coordinates of the ground stations whose data we use are given in Table 2. To identify the pulsations in satellite and terrestrial observations, we have removed the longperiod part - bay-like disturbances with a period $T \geq$ $60 \mathrm{~min}$, using a digital filter in the Matlab package (filtfilt.m, [https://www.mathworks.com/help/signal/ref/ filtfilt.html]), which does not introduce phase shifts in the output signal relative to the input one.

Table 1

Satellite coordinates in the interplanetary medium and magnetosphere

\begin{tabular}{|l|c|c|c|c|}
\hline \multirow{2}{*}{ Satellite } & \multirow{2}{*}{ Time, UT } & \multicolumn{3}{|c|}{ Coordinates } \\
\cline { 3 - 5 } & & $\mathrm{X}_{\mathrm{GSM}}, R_{\mathrm{E}}$ & $\mathrm{Y}_{\mathrm{GSM}}, R_{\mathrm{E}}$ & $\mathrm{Z}_{\mathrm{GSM}}, R_{\mathrm{E}}$ \\
\hline ACE & $11: 00$ & 237.10 & -22.08 & 31.31 \\
\hline WIND & $11: 00$ & 194.61 & -0.31 & -8.79 \\
\hline GOES 13 & $12: 00$ & 1.82 & -6.08 & 1.84 \\
\hline GOES 14 & $12: 00$ & -1.31 & -6.46 & 0.53 \\
\hline GOES 15 & $12: 00$ & -4.19 & -5.03 & -0.93 \\
\hline THEMIS E & $12: 00$ & -2.77 & -8.90 & 0.11 \\
\hline
\end{tabular}
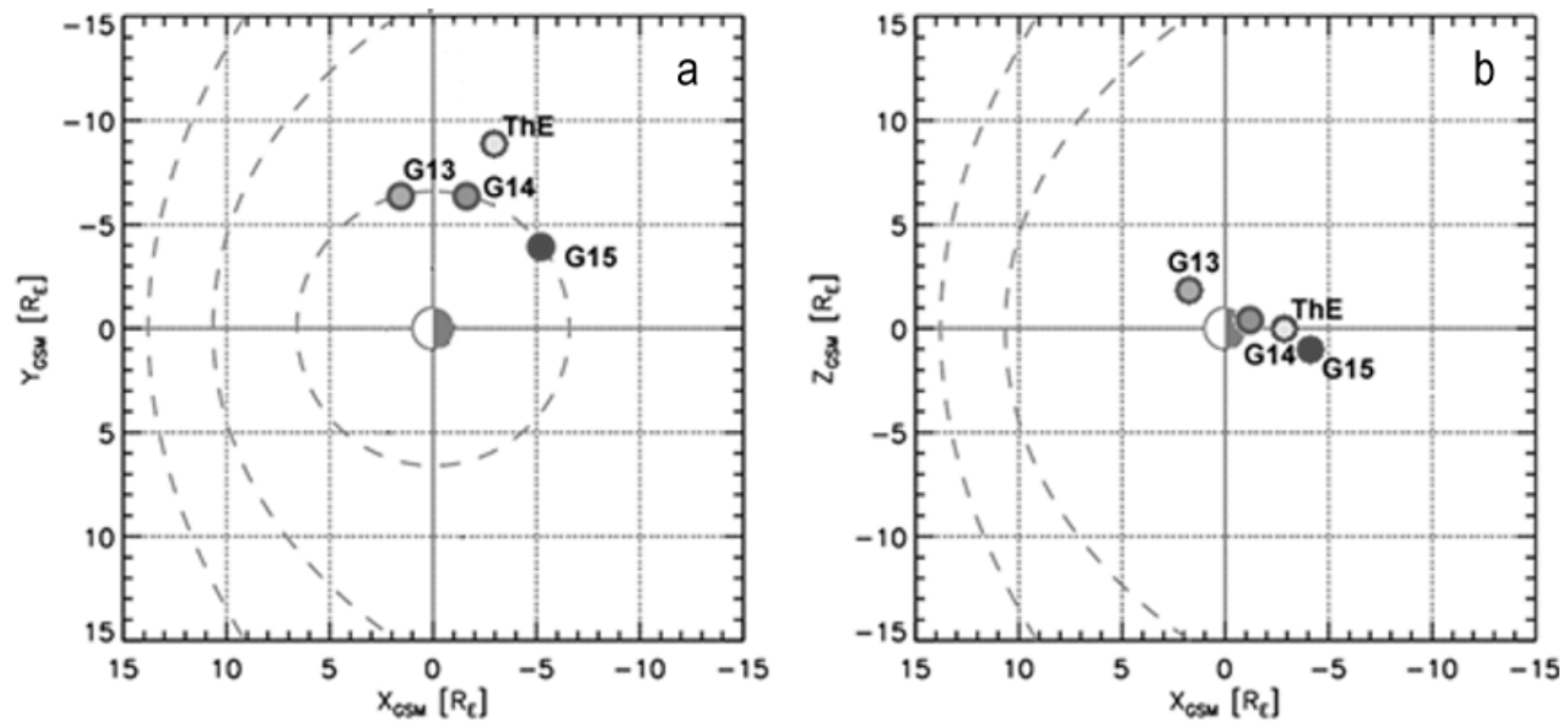

Figure 1. Location of different satellites in the XY $(a)$ and XZ (b) planes in the GSM coordinate system at 12.00 UT 
Table 2

Coordinates of geophysical (magnetic and riometric) stations

\begin{tabular}{|c|c|c|c|c|c|c|}
\hline \multirow{2}{*}{ Station } & \multirow{2}{*}{ Station code } & \multicolumn{2}{|c|}{ Geographic } & \multicolumn{2}{|c|}{ Geomagnetic } & \multirow{2}{*}{$\begin{array}{l}\text { Midnight } \\
\text { MLT, UT }\end{array}$} \\
\hline & & Latitude & Longitude & Latitude & Longitude & \\
\hline Yellowknife & YKC & 62.48 & 245.52 & 69.42 & 303.15 & $8: 22$ \\
\hline Fort Simpson & FSP & 61.76 & 238.77 & 67.47 & 295.11 & $8: 59$ \\
\hline Inuvik & INK & 68.25 & 226.70 & 71.50 & 276.95 & $10: 13$ \\
\hline Fort Yukon & FYU & 66.57 & 214.70 & 67.65 & 267.17 & $11: 00$ \\
\hline Deadhorse & DED & 70.36 & 211.21 & 70.87 & 260.73 & $11: 37$ \\
\hline Barrow & BRW & 71.30 & 203.25 & 70.60 & 253.43 & $12: 12$ \\
\hline Kotelnyy & KTN & 75.94 & 137.71 & 70.98 & 202.81 & $15: 50$ \\
\hline Dixon & DIK & 73.55 & 80.57 & 69.36 & 156.64 & $18: 15$ \\
\hline Eagle & EAG & 64.78 & 218.80 & 66.59 & 272.2 & $10: 37$ \\
\hline College & $\mathrm{CMO}$ & 64.87 & 212.14 & 65.45 & 266.18 & $11: 10$ \\
\hline Kenia College & T55 & 60.55 & 208.74 & 60.35 & 265.78 & $11: 02$ \\
\hline Shumagin & SHU & 55.38 & 199.54 & 53.29 & 260.26 & $11: 31$ \\
\hline Dawson City & DAW & 64.05 & 220.89 & 66.24 & 274.66 & $10: 24$ \\
\hline Fort Simpson & FSP & 61.76 & 238.77 & 67.47 & 295.11 & $08: 59$ \\
\hline Fort Smith & FSM & 60.02 & 248.05 & 67.47 & 307.71 & $08: 25$ \\
\hline Rabbit Lake & RAB & 58.22 & 256.32 & 67.00 & 319.92 & $07: 33$ \\
\hline Gillam & GIL & 56.38 & 265.36 & 66.16 & 333.92 & $06: 33$ \\
\hline Rankine Inlet & RAN & 62.82 & 267.89 & 72.45 & 336.88 & $06: 28$ \\
\hline Fort Churchill & FCC & 58.76 & 265.92 & 68.50 & 334.43 & $06: 31$ \\
\hline Island Lake & ISL & 53.86 & 265.34 & 63.70 & 334.21 & $06: 30$ \\
\hline Kiana & KIAN & 67.00 & 199.60 & 65.62 & 254.45 & $12: 02$ \\
\hline Cape Schmidt & CPS & 68.88 & 180.55 & 65.37 & 239.02 & $13: 12$ \\
\hline Chokurdakh & CHD & 70.62 & 147.89 & 65.67 & 214.11 & $15: 09$ \\
\hline Tixie & TIX & 71.58 & 129.00 & 66.70 & 198.71 & $16: 04$ \\
\hline Amderma & AMD & 69.50 & 61.40 & 65.85 & 138.20 & 19:09 \\
\hline Zhigansk & ZGN & 66.75 & 123.26 & 62.01 & 195.44 & $16: 13$ \\
\hline Zyryanka & ZYK & 65.75 & 150.78 & 60.54 & 218.76 & $14: 51$ \\
\hline Yakutsk & YAK & 60.02 & 129.72 & 54.88 & 202.6 & $15: 50$ \\
\hline
\end{tabular}

\section{RESULTS OF OBSERVATIONS}

\subsection{Variations of interplanetary medium pa-} rameters

Figure 2 presents ACE spacecraft (S/C) measurements of interplanetary medium parameters with a shift of $\sim 60 \mathrm{~min}$, which corresponds to the time of propagation of a disturbance from $\mathrm{S} / \mathrm{C}$ to a subsolar point at the magnetopause. Panel $a$ in the GSM coordinate system shows variations in the IMF $B_{z}$ component and $B$ modulus (panel $b$, right scale); panels $b-d$ depict variations in SW density $N$, velocity $V_{x}$, dynamic pressure $P_{\mathrm{d}}$. The Figure also presents variations in the $E_{y}$ component of the SW electric field, geomagnetic indices $A U, A L$ (panel $e-f$ ) at $05.00-15.00$ UT on December 08, 2017. The $B_{z}$ variations at steady southward IMF are seen to lead to intense fluctuations of the $A L$ index with amplitude 100-200 nT and a period 20-30 min, with maximum $A L$ 
being as great as $-370 \mathrm{nT}$. Note that this event was recorded in the absence of a magnetic storm, but during the decay phase of high speed SW stream. Next we will analyze the disturbances observed in the interval 10.00 15.00 UT, marked with a black rectangle at the bottom.

Comparison of variations in high-latitude geomagnetic activity indices with variations in $E_{y}$, calculated from the formula $E_{y}=V_{x} B_{y z} \sin ^{2}(\theta / 2)$, where $B_{y z}=\left(B_{y}{ }^{2}+B_{z}{ }^{2}\right)^{1 / 2}$, shows that they are similar (gray dashed lines on panels $e, f$ ). This fact and steady southward IMF $B_{z}$ suggest that the observed disturbances can be attributed to the so-called steady magnetospheric convection (SMC) disturbances [Pytte et al., 1978; Sergeev et al., 1996].

As in the events discussed in [Moiseev et al., 2016; Parkhomov et al., 2018], in our event there is anticorrelation between the SW density and the IMF modulus (Figure 2,b). It is more clearly shown in inset b1 from WIND S/C data. At the same time, the observed simultaneous increase in SW density and velocity resulted in a slight gradual increase in its dynamic pressure in the period considered.

\subsection{Observations of pulsations on Earth and in the magnetosphere}

Figure 3 illustrates variations in the geomagnetic field $H$ and $D$ components in the period 10.00-15.00 UT on December 08, 2017, according to data from highlatitude stations on the night side of Earth. The stations are located along high $\Phi^{\prime}=68^{\circ}-72^{\circ}$ (panels $a, b$ ) and lower $\Phi^{\prime}=63^{\circ}-66^{\circ}$ latitudes (panels $c, d$ ), where $\Phi^{\prime}$ is the geomagnetic latitude. The 15-30 min irregular pulsations were recorded from 10.50 to $13.30 \mathrm{UT}$ at auroral latitudes and clearly manifested themselves in the $D$ component of the geomagnetic field in the sector of 20 MLT with maximum amplitude to $100 \mathrm{nT}$.

On the left panel, for the BRW and DED stations in the $H$ component we can see pulsations that started and ended earlier than in the $D$ component. Note that the pulsations were recorded during a DP2-type disturbance characterized by enhancement of the westward electrojet (negative magnetic bay in the $H$ component, panel $a$ ) in the dawn side and the eastward electrojet (positive magnetic bay, panel $c$ ) in the dusk side.

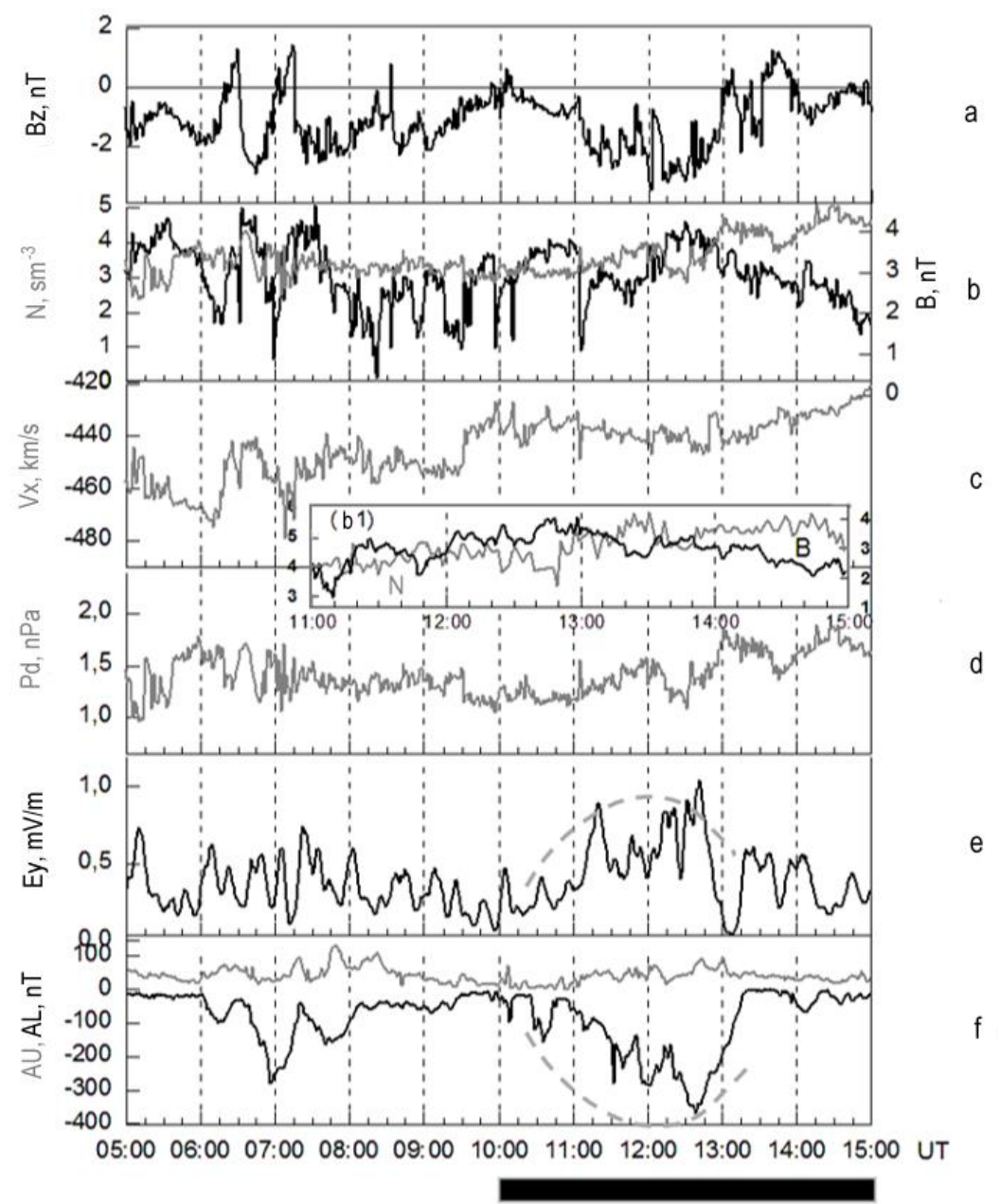

Figure 2. Time dependence of variations in interplanetary medium parameters according to ACE S/C data: IMF $B_{z}(a)$, SW density $N$ (gray line, left scale), IMF modulus $B$ (black line, right scale) $(b)$, SW velocity component $V_{x}(c)$, SW dynamic pressure $P_{\mathrm{d}}(d), E_{y}$ component of the SW electric field $(e), A U$ and $A L$ geomagnetic activity indices $(f)$ at $05-15 \mathrm{UT}$. The ACE S/C data is given with a shift of $\sim 60 \mathrm{~min}$. Inset b1: plasma density variations (gray line, left scale) and the IMF modulus $B$ (black line, right scale) obtained from WIND measurements 
a

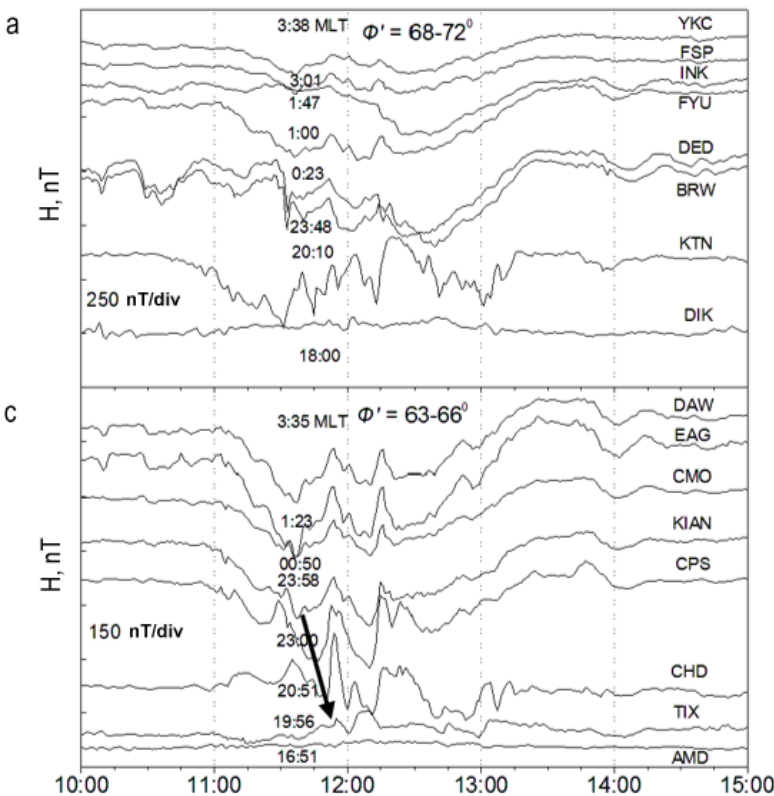

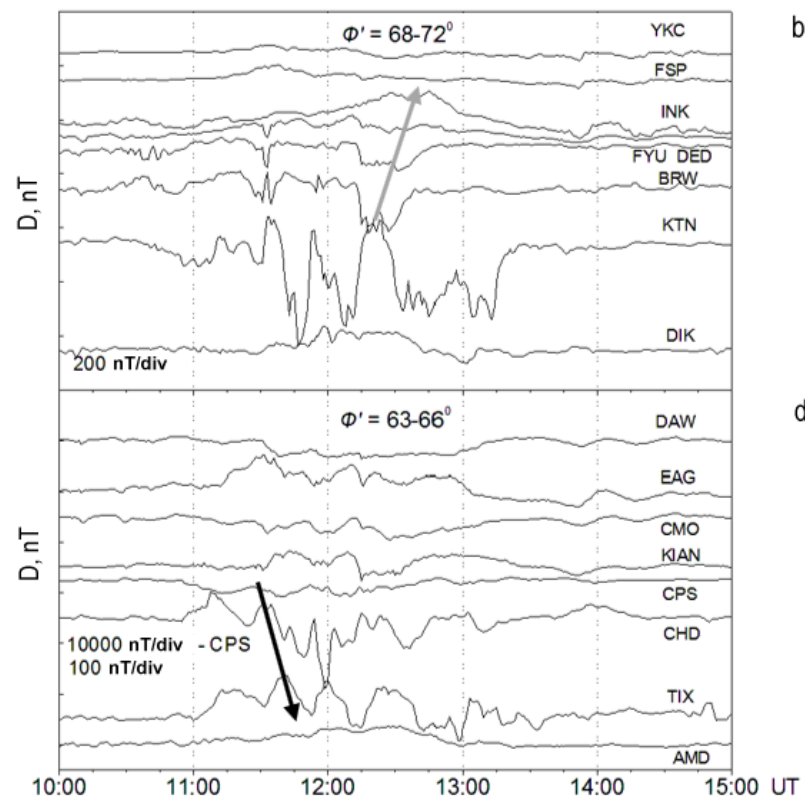

Figure 3. Variations in geomagnetic field $H$ and $D$ components at geomagnetic latitudes $68^{\circ}-72^{\circ}(a, b)$ and $63^{\circ}-66^{\circ}(c, d)$ as a function of time. Arrows indicate direction of propagation of geomagnetic pulsations to the east in the post-midnight sector and to the west in the pre-midnight sector. On panels $a, c$ is the MLT sector of stations

Phase delays in Pi3 pulsations at the stations in different MLT sectors indicate azimuthal propagation of the pulsations (schematically shown by arrows) from the midnight to the east in the post-midnight sector and to the west in the pre-midnight sector at a velocity $1-6 \mathrm{~km} / \mathrm{s}$.

Figure 4 shows variations in the geomagnetic field $H$ and $D$ components along magnetic meridians $00-01$ MLT (post-midnight sector, panels $a, b$ ) and 20-21 MLT (pre-midnight sector, panels $c, d$ ) at geomagnetic latitudes from $70^{\circ}$ to $60^{\circ}$. Bottom panels $(e, f)$ demonstrate variations in the geomagnetic field $Z$ and $H$ components at several stations in these sectors. Referring to Figures 3 and $4, a, c$, in the post-midnight sector a westward electrojet was recorded; whereas in the premidnight sector, an eastward one. The pulsations had the form of negative variations in the post-midnight sector and positive variations in the pre-midnight sector. From variations in the $Z$ (black solid curve) and $H$ components (gray dashed curve) (Figure 4,e) it follows that the center of gravity of the eastward electrojet was in the pre-midnight sector to the north of the KTN station $\left(70.98^{\circ}\right)$, and the center of gravity of the westward electrojet in the post-midnight sector was at the latitude of the FYU station (Figure 4, $f$ ), which corresponds to the geomagnetic latitude of $67.6^{\circ}$. It is evident that there were considerable variations in the $Z$ component relative to zero at this station.

Arrows in Figure 4, $d$ indicate phase delays suggesting that the magnetic pulsations propagate to the equator. Analysis of propagation velocities was conducted using the method described in [Makarov et al., 2002] and was applied to longitude and latitude variations (Figures 3, 4), with trends of bay-like disturbances removed. These filtered variations denoted by $\delta H$ and $\delta D$ are shown in Figures 5 and 6 respectively. Propagation directions are indicated by arrows: solid arrows mark azimuthal propagation (from the midnight to the east and west); dashed arrows, meridional propagation (to the equator). Values of the propagation velocities are listed in Table 3 .

Figure 7 shows phase delays during recording of 95 $\mathrm{keV}$ proton $(a)$ and $150 \mathrm{keV}$ electron $(b)$ fluxes from satellites GOES 13 (G13), GOES 14 (G14), and GOES 15 (G15), as well as phase delays in riometric absorption variations in azimuthal $(c)$ and meridional directions $(d)$. Note that unlike electrons, the bay-like disturbance hardly manifested itself in proton fluxes. However, if electron fluxes are higher in G15 located closer to the midnight sector, proton fluxes are higher in G13 located closer to the dawn sector. This can be explained by the drift of the protons to the west, and the electrons to the east.

The profile of riometric absorption of cosmic radio noise in the DAW station at 01.36 MLT coincides with the change in the electron flux in G15 almost without delay. This suggests that electron injections contribute significantly to the observed riometric absorption variations. From the relative delays in variations of particle fluxes we estimated disturbance propagation velocities, which were $60-80 \mathrm{~km} / \mathrm{s}$ for the electron fluxes, as derived from G15, G14, G13 observations at 04.00-06.00 MLT. Azimuthal propagation of the electron fluxes coincides in direction with propagation of riometric absorption (Figure 7,c). We have compared the riometric absorption dynamics with geomagnetic field $\delta D$ component variations at the same stations shown in Figure $8, c, d$. In this case, the $\delta D$ component has dynamics similar to that of riometric absorption in both azimuthal and meridional directions. The velocities estimated from riometric data are also listed in Table 3 . We can see that in the pre-midnight sector positive azimuthal velocities corresponding to westward propagation are recorded; in the post-midnight sector, negative velocities corresponding to eastward propagation. The azimuthal velocity $V_{\text {azim }}$ increases in the pre- and post-midnight sectors with distance from the midnight meridian; in 18-19 MLT sectors at a latitude of $70^{\circ} V_{\text {azim }}=16.5 \mathrm{~km} / \mathrm{s}$; at a latitude of $66^{\circ}, V_{\mathrm{ds}}=9.4 \mathrm{~km} / \mathrm{s}$. In the $02.00 \mathrm{MLT}$ sector at 

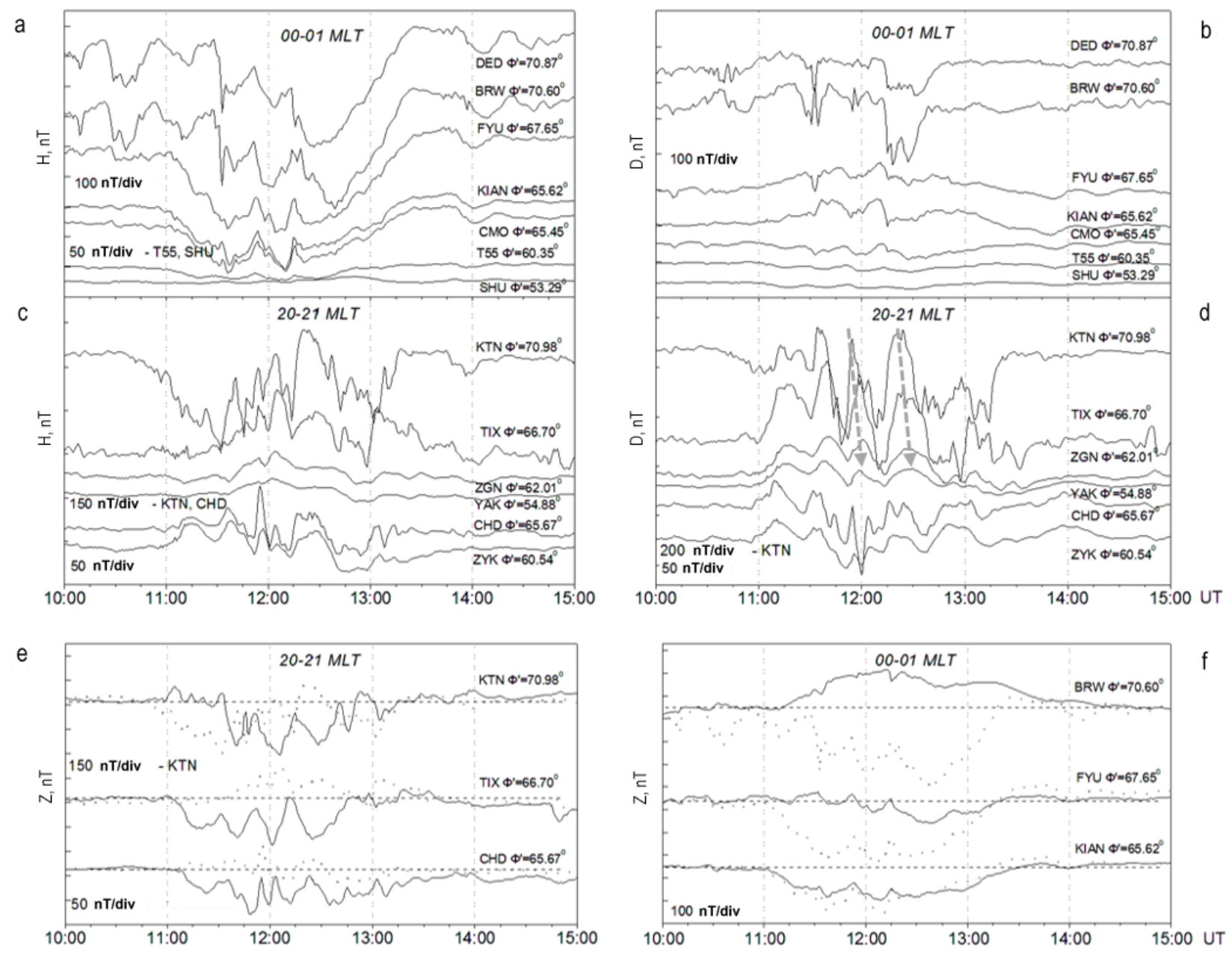

Figure 4. Time dependence of variations in the geomagnetic field $H$ and $D$ components along geomagnetic meridians $00-01$ MLT $(a, b)$ and 20-21 MLT $(c, d)$, variations in the $Z$ and $H$ components indicated by solid black and gray dashed curves respectively at certain stations of the meridian 20-21 MLT $(e)$ and 00-01 MLT $(f)$
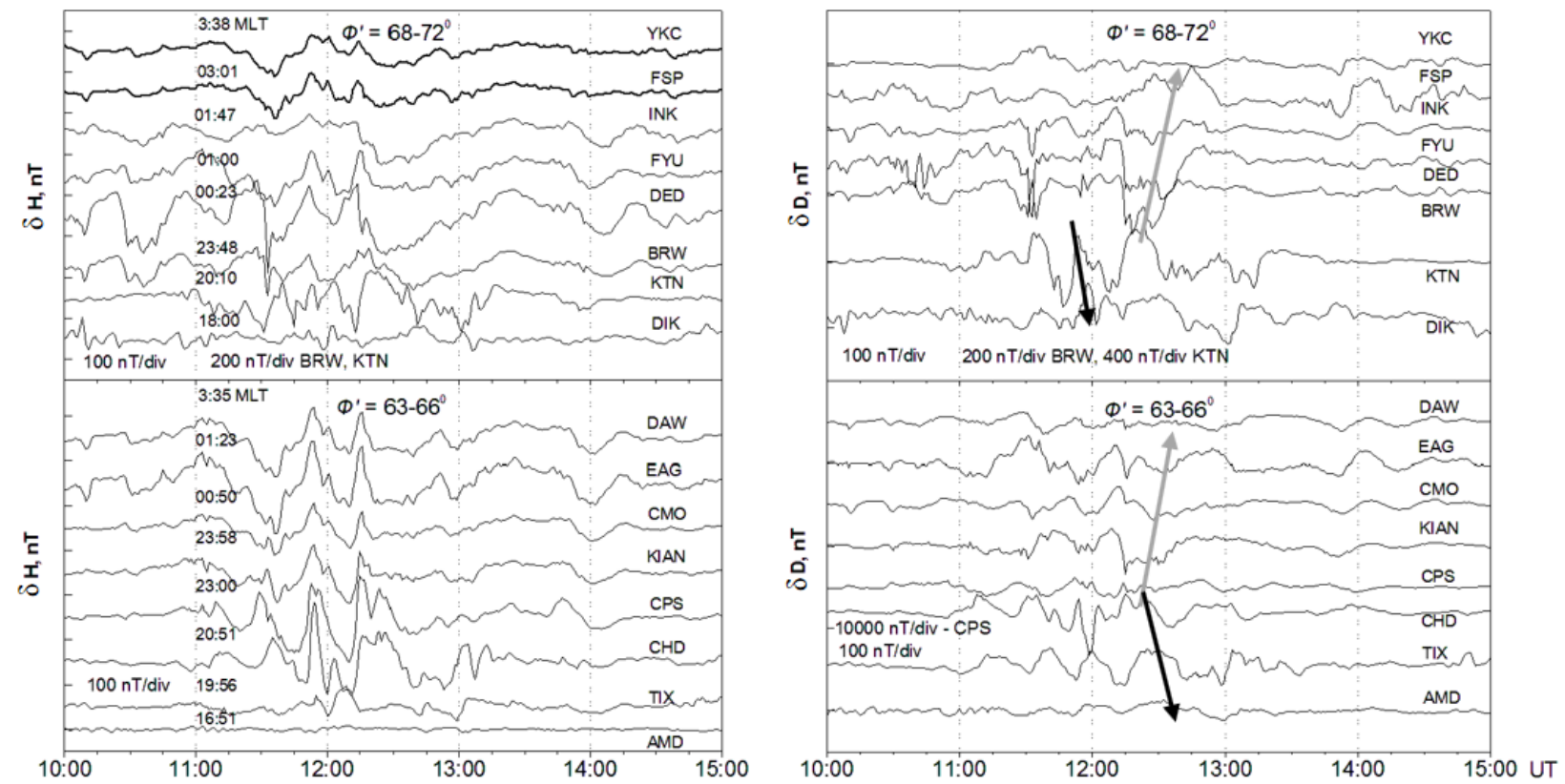

Figure 5. Variations in the geomagnetic field $\delta H$ and $\delta D$ components filtered from the original dataset over time from measurements at the same stations as in Figure 3. Arrows indicate the direction of propagation of geomagnetic pulsations to the east in the post-midnight sector and to the west in the pre-midnight sector. Filter cutoff period $T \geq 60 \mathrm{~min}$ 

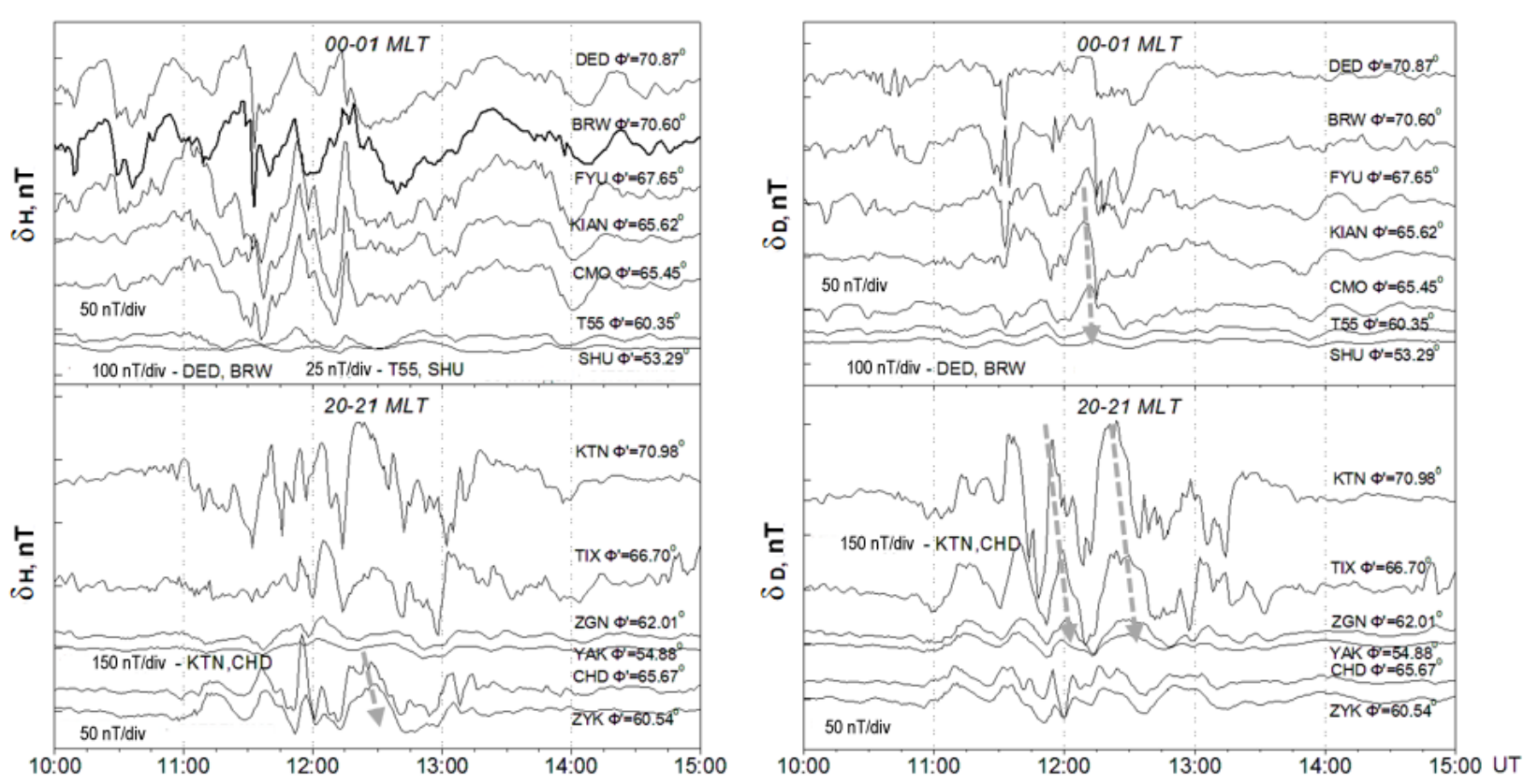

Figure 6. Variations in the geomagnetic field $\delta H$ and $\delta D$ components filtered from the original dataset as a function of time along geomagnetic meridians 00-01 MLT (top panel) and 20-21 MLT (bottom panel). Arrows indicate equatorward propagation of pulsations. Filter cutoff period $T \geq 60 \mathrm{~min}$

Table 3

Value and direction of propagation velocities in the ionosphere according to data from ground-based magnetometers and riometers. Negative azimuthal velocities correspond to eastward propagation; positive ones, to westward propagation.

Positive meridional velocities correspond to equatorward propagation

\begin{tabular}{|c|c|c|c|c|c|c|}
\hline \multirow{3}{*}{ Instruments } & \multicolumn{6}{|c|}{ Propagation } \\
\hline & \multicolumn{3}{|c|}{ Azimuthal } & \multicolumn{3}{|c|}{ Meridional } \\
\hline & $\begin{array}{l}\text { Velocities, } \\
\mathrm{km} / \mathrm{s}\end{array}$ & $\begin{array}{l}\text { Geomagnetic } \\
\text { latitude, }{ }^{\circ}\end{array}$ & MLT, hr:min & $\begin{array}{c}\text { Velocities, } \\
\mathrm{km} / \mathrm{s}\end{array}$ & $\begin{array}{l}\text { Geomagnetic } \\
\text { latitude, }{ }^{\circ}\end{array}$ & MLT, hr:min \\
\hline \multirow{14}{*}{ 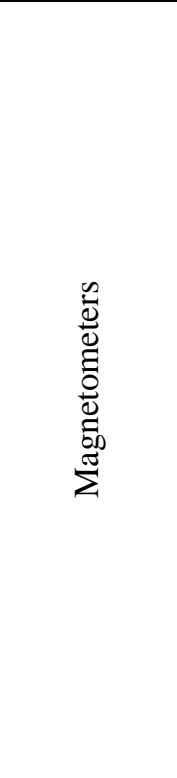 } & -6.55 & 69.49 & $2: 00$ & 0.69 & 67.71 & \multirow{2}{*}{$05: 30$} \\
\hline & -0.88 & 69.26 & $1: 30$ & 0.49 & 66 & \\
\hline & -0.93 & 70.74 & 1:00 & 1.82 & 69.13 & \multirow{5}{*}{$00: 00-01: 00$} \\
\hline & -0.62 & 69.58 & $0: 40$ & 3.13 & 66.64 & \\
\hline & 16.54 & 70.17 & 18:40 & 1.7 & 66.55 & \\
\hline & -2.52 & 66.02 & $1: 10$ & 7.87 & 62.9 & \\
\hline & -3 & 65.54 & $0: 20$ & 7.27 & 56.82 & \\
\hline & 10.62 & 65.52 & $21: 50$ & 1.89 & 68.84 & \\
\hline & 1.6 & 66.19 & $20: 20$ & 4.83 & 64.36 & $20: 00-21: 00$ \\
\hline & 9.4 & 66.28 & 18:00 & 3.96 & 63.11 & \\
\hline & -2.6 & 66.4 & $5: 00$ & - & - & - \\
\hline & -3.63 & 67.4 & 4:00 & - & - & - \\
\hline & -3.05 & 67.9 & $3: 17$ & - & - & - \\
\hline & -0.93 & 66.85 & $2: 15$ & - & - & - \\
\hline & -0.86 & 66.4 & 5:00 & -0.1 & 67.71 & 05:30 \\
\hline$\frac{\overline{0}}{0}$ & -0.52 & 67.4 & 4:00 & - & - & - \\
\hline$\frac{\tilde{\sigma}}{2}$ & -1.63 & 67.9 & $3: 17$ & - & - & - \\
\hline & -2.4 & 66.85 & $2: 15$ & - & - & - \\
\hline
\end{tabular}


a latitude of $69.5^{\circ}$, the velocity increases to $-6.5 \mathrm{~km} / \mathrm{s}$ (the sign "-" indicates eastward propagation). The azimuthal velocity in the post-midnight sector 2-5 MLT at latitudes $66.4-67.9 V_{\text {azim }}=0.9-3.6 \mathrm{~km} / \mathrm{s}$ as derived from magnetometer data, whereas according to riometer data it is several times lower $-V_{\mathrm{ds}}=0.5-0.7 \mathrm{~km} / \mathrm{s}$. The velocity of equatorward meridional propagation $V_{\text {mer }}$ in the 20-21 MLT sector also increases with decreasing latitude from $1.9 \mathrm{~km} / \mathrm{s}$ at a latitude of $69^{\circ}$ to $4-4.8 \mathrm{~km} / \mathrm{s}$ at a latitude $63^{\circ}-64^{\circ}$. Such dynamics is also observed in the post-midnight sector 00-01 MLT, where the equatorward propagation velocity increases from $V_{\text {mer }}=1.8$ $\mathrm{km} / \mathrm{s}$ at a latitude of $69^{\circ}$ to $V_{\text {mer }}=7.8 \mathrm{~km} / \mathrm{s}$ at a latitude of $63^{\circ}$. In the dawn sector $05.30 \mathrm{MLT}$, the southward velocity was $0.75 \mathrm{~km} / \mathrm{s}$ according to magnetometer data and about 7 times lower according riometer data. The lower velocities from riometer data as compared to those from magnetometer data may be attributed to different forms of disturbances recorded by these instruments. In the riometer, disturbances look smoother, and therefore it is possible to estimate time delays between the main maxima (minima) of bay-like (slow) disturbances. Spanswick et al. [2005] have carried out a statistical analysis of propagation of substorm injections in azimuthal and meridional directions, using riometer observations. For the analysis, the authors took, among others, data from some stations presented in Figures 7, 8. They compared azimuthal propagation velocities, derived from magnetometer and riometer data, and obtained similar values $(2-3 \mathrm{~km} / \mathrm{s})$. As for meridional propagation, the authors recorded only pole propagation. Thus, in our event the riometer data allows us to compare only propagation direction, not its velocity, with that obtained from magnetometer observations.

Figure 9 shows electron density variations compared with variations in the $B$ modulus and $B_{z}$ component of the geomagnetic field, measured by the ThE satellite $(a)$. On lower panels are variations in electron $(b)$ and protons $(c)$ fluxes in the same satellite.

The similarity in the behavior of $B$ and $B_{z}$ suggests that the full vector varies along the $z$ coordinate, which corresponds to compression field variations, as shown in [Li et al., 2011] from ThE satellite measurements. Of note also is that the $n$ and $B$ variations were recorded in antiphase. Such $n$ and $B$ variations are typical of slow magnetosonic (SM) waves [Van de Hulst, 1951] or mirror mode oscillations [Hasegawa, 1969; Woch et al., 1988] and also indicate the compression nature of oscillations in the magnetosphere. In [Mager et al., 2013; Kostarev, Mager, 2017], compression small-scale oscillations are interpreted using the drift compression wave mode, which also features opposite variations of $n$ and $B$. However, this mode is recorded mainly during the magnetic storm recovery phase.

The electron fluxes in the interval of interest showed dispersionless injection. From the similarity in forms of electron fluxes with $E=139 \mathrm{keV}$ in ThE and lowerenergy particles $(E=95 \mathrm{keV})$ in G14 (Figure 7), and from the closest values of the $z$ coordinate of these satellites (Figure 1), we have estimated the radial velocity of electron flux propagation between these satellites (along the $x$ coordinate, the distance between the satellites was $8929.2 \mathrm{~km}$ ) at $113 \mathrm{~km} / \mathrm{s}$.

It should be noted that in the interval 11.15-12.15 UT there was a slight increase in the proton variations followed by generation of 10-15 min pulsations. Amplitude of the pulsations peaked in the $15.7 \mathrm{keV}$ proton flux (gray color in Figure 9,c). These pulsations, like electron density variations, were recorded in antiphase with the magnetic field, but they had a more regular form.

Figure 10, $a-c$ shows $V_{x}, V_{y}, V_{z}$ components of the electron velocity measured by the ThE satellite. Panel $d$ depicts variations in electron $\delta n$ and magnetic field $\delta B$ densities in the ThE satellite. Panel $e$ illustrates IMF $\delta B_{z}$ variations from the ACE satellite and $\delta V_{x}$ velocity component from ThE. From data, presented on panels $d, e$, we excluded long-period bay-like disturbances, using a filtering method. We can see that in the ThE satellite as well as in ground stations in this sector, eastward radial propagation of electrons occurred on the night side. We evaluated the horizontal velocity component $V_{\text {hor }}=\left(V_{x}\right.$ $\left.{ }^{2}+V_{y}^{2}\right)^{1 / 2}=127 \mathrm{~km} / \mathrm{s}$ at $12 \mathrm{UT}$. At the same time, the electron velocity had an equatorward vertical component $V_{z}=-357 \mathrm{~km} / \mathrm{s}$, which was more than twice higher than the horizontal component. Both azimuthal and meridional propagation agrees in general with ground-based data (Figures 7,8). Comparison between $\delta n$ and $\delta B$ (panel $b$ ) shows their closer correlation (anticorrelation) than in Figure 9. Comparison of $V_{z}$ variations with $H$ variations at the RAN station (Figure $8, b$ ) points to their similarity. From this we can conclude that the satellite was near the equatorial projection of the field line of this station, and electron precipitation contributed significantly to the ground-observed geomagnetic field $H$-component variations.

\section{DISCUSSION}

Thus, in the event under study on Earth's night side there were geomagnetic Pi3 pulsations, which propagated in azimuthal and meridional directions and had a maximum intensity at latitudes of auroral electrojets of the DP2 current system. In the dawn-side magnetosphere, the ThE satellite from magnetometer and plasma detector data recorded the azimuthally small-scale oscillations that did not manifest themselves in ground-based magnetic field measurements. Manifestation of the Pi3 pulsations in magnetometer, riometer, and optical observations has also been examined in [Belakhovsky et al., 2015]. The authors observed a sharp increase in energetic particle fluxes from satellite observations, an increase in electron precipitation from riometer absorption data, and an enhancement of auroral intensity from all-sky camera data. The magnetic pulsations modulated electron fluxes and riometric absorption. There is no such modulation in our event, and riometer data shows only bay-like disturbances. This can be attributed to a small value of the compression component of the pulsations in the magnetosphere, which modulates the electroncyclotron instability increment [Coronity, Kennell, 1972]. 


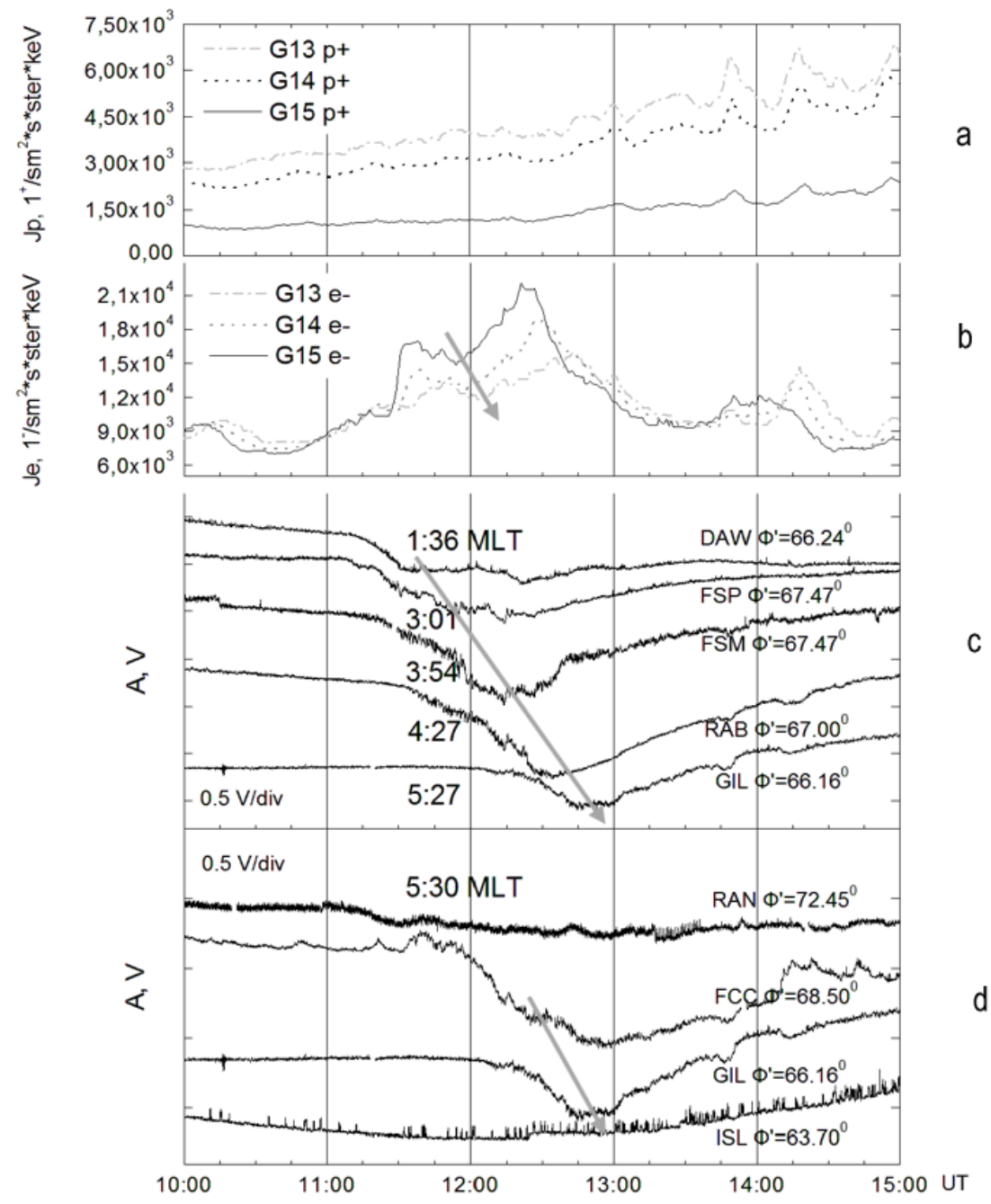

Figure 7. Time variations in $95 \mathrm{keV}$ proton fluxes $(a)$ and $150 \mathrm{keV}$ electron fluxes $(b)$ in G13, G14, G15 satellites; riometric absorption variations in azimuth at stations located along the geomagnetic latitude $66^{\circ}-67.5^{\circ}(\mathrm{c})$ and along the meridian of $5: 30$ $\operatorname{MLT}(d)$
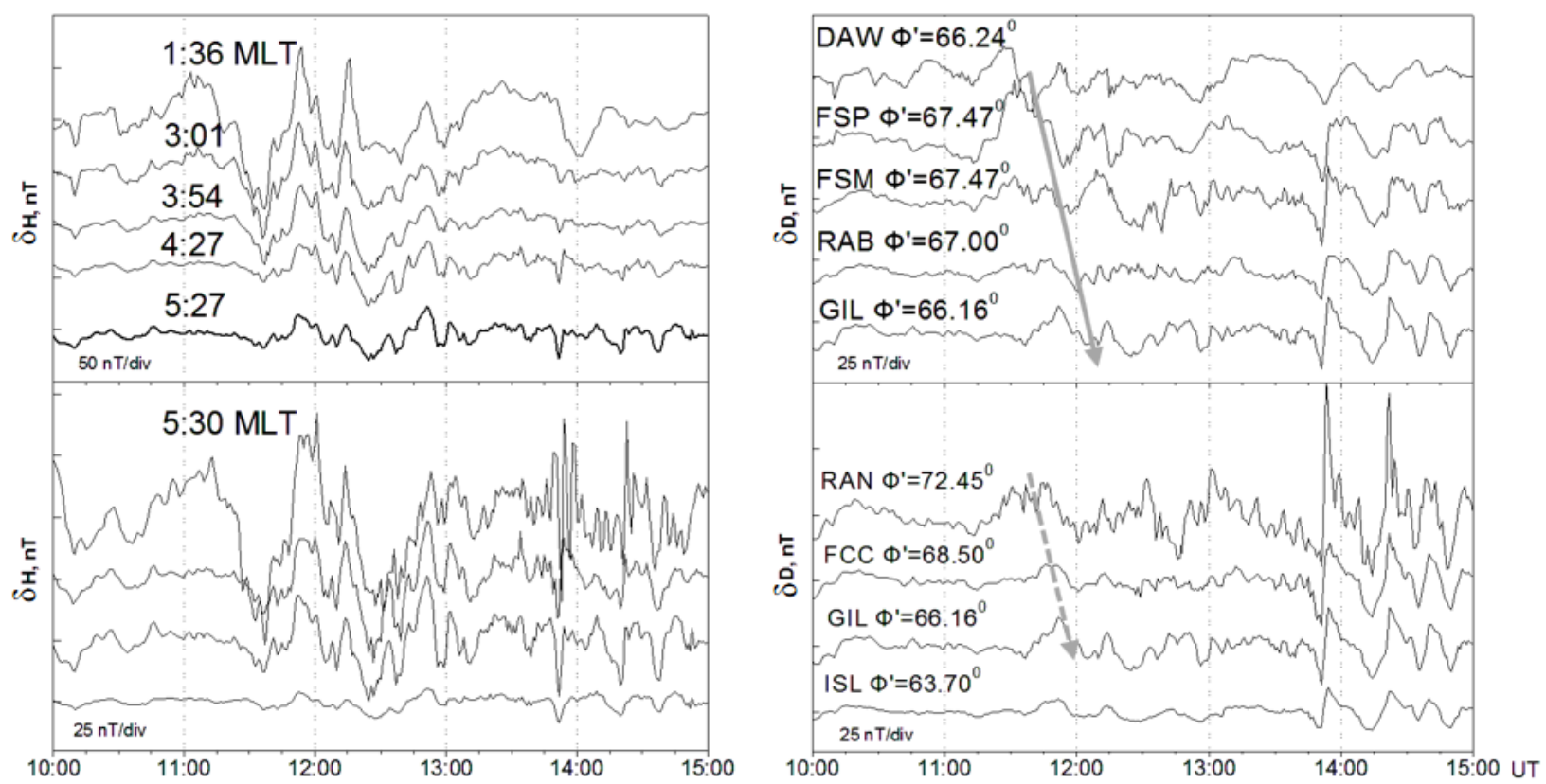

Figure 8. Variations in the geomagnetic field $\delta H(a, b)$ and $\delta D(c, d)$ components as a function of time at the same stations as in Figure 7 


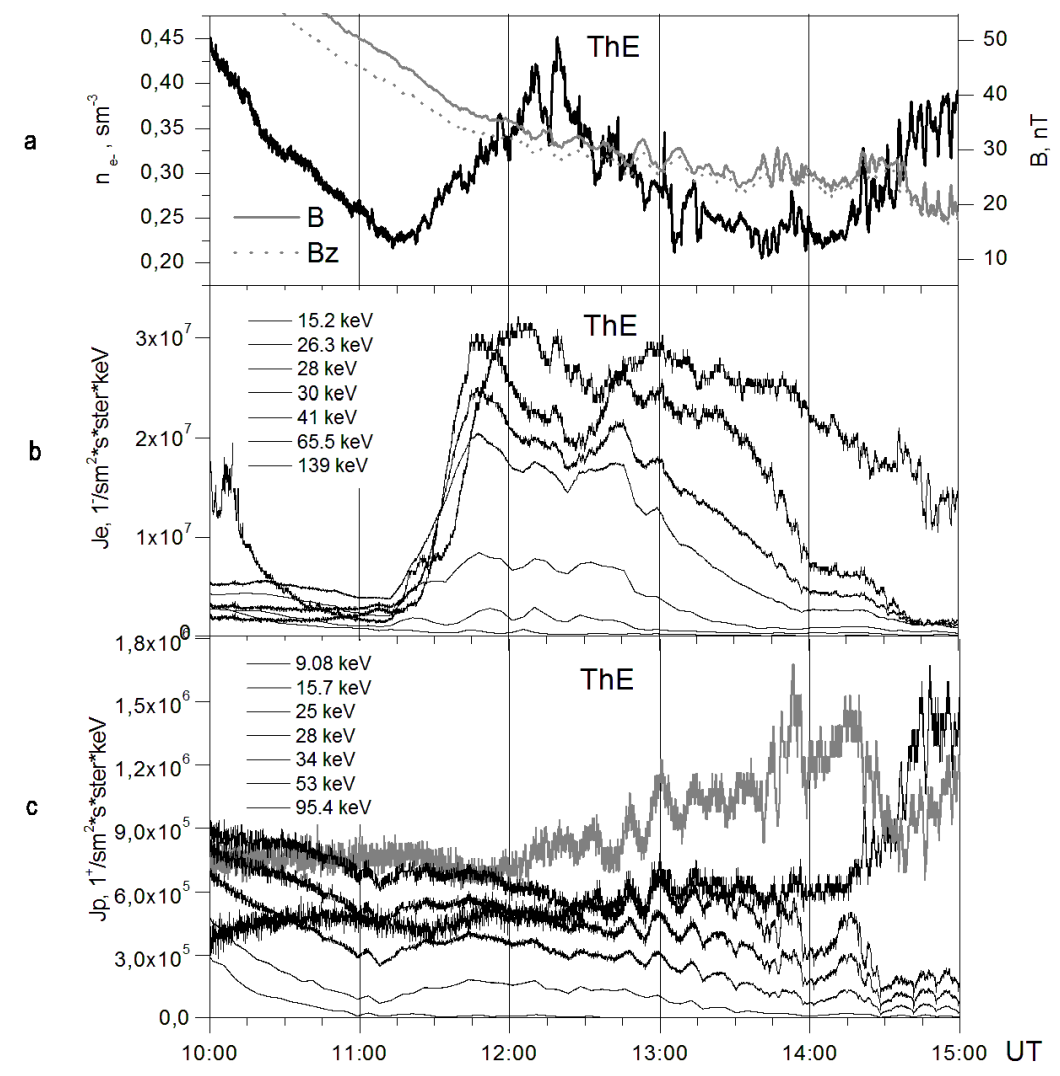

Figure 9. Variations in the electron density $n$ (black line, left scale), modulus $B$ and $B_{z}$ component of the geomagnetic field (gray line, right scale) $(a)$; variations in energetic electron $J_{\mathrm{e}}(b)$ and ion $J_{\mathrm{p}}(c)$ fluxes as a function of time from ThE measurements

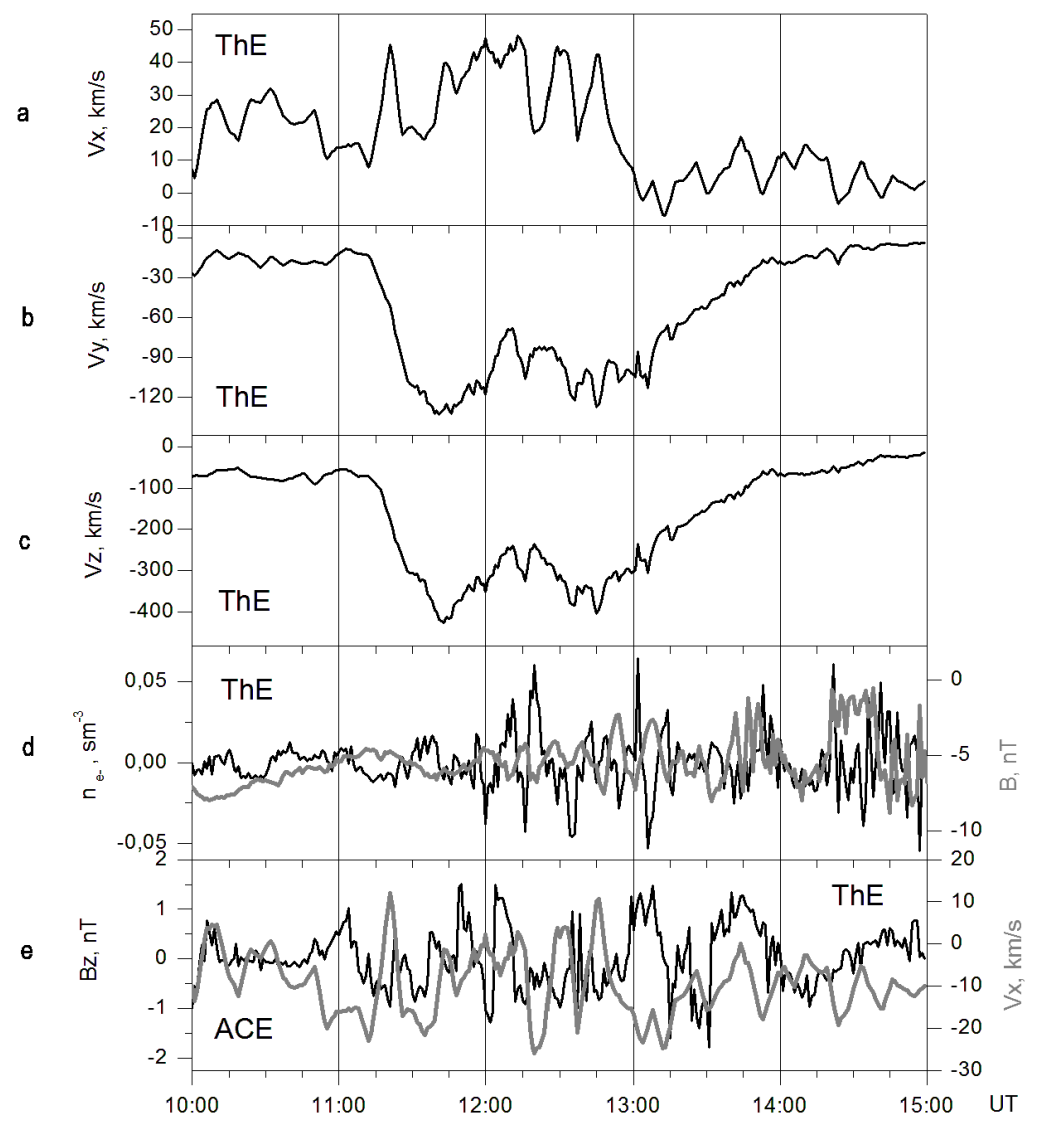

Figure 10. $V_{x}, V_{y}, V_{z}$ components of electron velocity $(a-c)$, electron density and magnetic field from ThE satellite measurements $(d)$, IMF $B_{z}$ component and electron velocity $V_{x}$ component $(e)$ from ACE S/C data as a function of time 


\subsection{Propagation of pulsations in the iono- sphere and inner magnetosphere}

According to [Kiselev, Raspopov, 1976 ] and [Saito, 1978], a subclass of Pi3 pulsations are Ps6 pulsations. Their peculiarity is high intensity in the $D$ and $Z$ components as compared to the $H$ component. Ps6 are generally recorded at night and dawn [Saito, 1978; Rostoker, Barichello, 1980] concurrently with polar auroras recorded during a substorm (auroral torch). However, there were no auroras in the event of interest. Thus, we can assume that the Pi3 pulsations in our event have some features typical of Ps6 pulsations (manifestation in the $D$ and $Z$ components), and may indicate the contribution of field-aligned currents to generation of these pulsations. The pulsations, according to the localization of their amplitude at latitudes close to the position of convection electrojets, are driven by their oscillations.

It is common knowledge that wave disturbances propagate along the azimuth and meridian, with the propagation direction dependent on their source: antisunward propagation is usually defined by external sources, e.g. by Kelvin-Helmholtz instability or by SW dynamic pressure pulses $P_{\mathrm{d}}$ [Mishin, 1993]; whereas sunward propagation, by the internal source. However, as shown in [Klibanova et al., 2014; Moiseev et al., 2016], the external and internal sources may operate simultaneously, thus causing wave disturbances to propagate in opposite direction in different regions of the magnetosphere. Almost all studies that analyze wave disturbances from ground-based data mention the fact of their meridional propagation to the magnetic pole. The main source of disturbances in this case is the fast MS wave - Alfvén wave conversion along field lines of different lengths and a delay in the arrival of the Alfvén wave when it propagates along longer field lines [Parkhomov et al., 2005]. Opposite propagation of wave disturbances to the equator is examined in papers that analyze radar measurement data indicating a relationship of such waves with disturbances in the ionosphere F-region and their small-scale source. Thus, Yeoman et al. [1992] have used SABRE radar data to describe equatorward propagation in the dusk sector. The authors suggested that such propagation is driven by the wave - particle interaction, namely the interaction between ring current hot protons and the plasmapause. We have not come across papers that mention equatorward propagation of wave disturbances identified from ground-based magnetometer data. Lack of ground-based magnetometer data on such waves may be associated with the fast decay of waves recorded by a radar (see [Yeoman et al., 1992]).

It is interesting to see how the propagation velocity varies with latitude. If we assume that the observed signal phase delays are caused by wave propagation, then, according to theoretical concepts, due to the large difference in the Alfvén velocity at the point of reconnection, MHD waves move to Earth at the velocity that is by an order of magnitude greater in tail lobes than in a denser plasma sheet. Comparing velocities obtained from magnetometer data at latitudes $69^{\circ}-70^{\circ}$ with velocities at latitudes $65^{\circ}-66^{\circ}$, we can conclude that ve- locities are higher at lower latitudes, i.e. the reverse is true. With this in mind, we can assume that in our event propagation depends on plasma injections in the region of convection electrojets, which change the value of ionospheric plasma conductivity, thus leading to the observed dynamics in the azimuthal and meridional directions.

The wave velocities, listed in Table 3 , in both meridians show a two-threefold increase at latitudes below $66^{\circ}$. Such dynamics of the meridional velocity can be explained by higher azimuthal propagation velocities at lower latitudes.

Note that the horizontal component of electron velocity $V_{\text {hor }}=127 \mathrm{~km} / \mathrm{s}$ in the ThE satellite is comparable with the injection propagation velocity $(113 \mathrm{~km} / \mathrm{c})$ between ThE and G14, which again emphasizes the significant contribution of electron injections to propagation of wave disturbances. This velocity is several times higher than that of substorm injections $(24 \mathrm{~km} / \mathrm{s})$ estimated in [Reeves et al., 1996] through the analysis of several events. This difference may be due to different positions of the satellites in their study and in our event.

While in this event we examine $\mathrm{Pi} 3$ pulsations under steady magnetospheric convection, the processes occurring in the magnetosphere-ionosphere system do not differ fundamentally from the substorm disturbances: the intensity of the eastward electrojet, as well as the duration of observation, is much lower than that of the westward one, dispersionless electron injections are similar to substorm ones. Furthermore, out of necessity to maintain the pressure balance, it is held that steady convection is hardly possible in the tail of the magnetosphere, and constant energy input must generate repetitive substorms. Solovyev et al. [1999] have examined similar disturbances on the night side in events driven by steady magnetospheric convection. These disturbances were accompanied by generation of convection electrojets and 5-15 min Ps6 pulsations. A distinctive feature of this event was the generation of N-S auroras, which expanded to the equator with $V \geq 1 \mathrm{~km} / \mathrm{s}$. A satellite that was on the night side detected earthward highspeed plasma flows. The authors suggested that the auroras in their event are an optical manifestation of highspeed plasma flows in the magnetotail.

Kozlovsky et al. [2001] have compared the equatorward movement of the auroral arc with ionospheric convection variations during the substorm. It has been found that the velocity of movement of the arc and the convection velocity are $0.4-0.6 \mathrm{~km} / \mathrm{s}$. The authors have attributed such dynamics of the auroral arc to IMF $B_{z}$ variations due to reconnection, which give rise to a dawn-dusk electric field in the nightside magnetosphere, which determines the earthward plasma motion manifesting itself in the equatorward movement of the arc.

In this event, the equatorward propagation velocities from magnetic and riometer observations are of the same order of magnitude as the arc movement velocity in the event discussed in [Kozlovsky et al., 2001] but closer to the aurora expansion velocity obtained in [Solovyev et al., 1999]. Equatorward propagation in our event occurs without considerable delay in the interval 
12-13 UT in different meridians: 00-01 MLT, 20-21 MLT, 05.30 MLT (Figures 6-8), which indicates a large-scale source of propagation. Comparison of equatorward propagation with the SW electric field dynamics $E_{y}$ (Figure 2) shows that $E_{y}$ increased from 0.2 to 1 $\mathrm{mV} / \mathrm{m}$ in the interval $10.15-12.45$ UT and equatorward propagation was observed from $11.30 \mathrm{UT}$ after $E_{y}$ had reached $0.5 \mathrm{mV} / \mathrm{m}$. Oscillations of the $V_{x}$ component in ThE in our event are similar to velocity variations in Geotail in the event discussed in [Solovyev et al., 1999], but their amplitude is lower by an order of magnitude. Moments of two minima in $V_{\mathrm{z}}$ in ThE are close to those of SW electric field maxima (11.20 UT and 12.40 UT, Figure. $2, e$ ). IMF $B_{\mathrm{z}}$ variations coincide in time and are close to the period of maximum variations of $V_{x}$ in Figure 10. Perhaps they modulate the intensity of the DP2 current system through ionospheric conductivity variations caused by modulation of earthward plasma velocity. Presumably the velocity variations and the magnetic field (density) variations in ThE reflect physical processes occurring in different magnetospheric regions. Velocity variations provide information about reconnection in the magnetotail. Pulsations in the magnetic field (density) are associated with the local process of wave particle interaction. The pulsations observed in ThE are small-scale since they are not recorded on Earth in this sector.

As mentioned above, the antiphase plasma density and magnetic field variations may correspond both to an SM wave, and to a mirror mode (MM) wave as Alfvén waves are not accompanied by density variations. SM waves are known to occur mainly in SW and magnetosheath; whereas MM waves, in the magnetosphere [Woch et al., 1990]. Pulsations in the dawn sector of the magnetosphere were recorded at $\beta>1$; however, values of the hydrodynamic criterion for an MM wave in our event $(\Gamma>0)$ do not correspond to the values necessary for the development of this instability: $\Gamma=1+\beta_{\text {per }}[1-$ $\left.T_{\mathrm{per}} / T_{\mathrm{par}}\right]<0 \quad$ [Hasegawa, 1969], where $\beta_{\mathrm{per}}=(n$ $\left.{ }_{\mathrm{i}} k T_{\mathrm{ipar}}+n_{\mathrm{e}} k T_{\mathrm{eper}}\right) /\left(B^{2} /\left(2 \mu_{0}\right)\right), T_{\text {par }}$, per are the plasma temperature parallel and perpendicular to the magnetic field, $\mu_{0}$ is the permeability of free space. Thus, the waves recorded in the magnetosphere are most likely to be SM waves.

\subsection{Penetration of MHD waves from the so- lar wind into the magnetosphere and their transformation}

Comparison of IMF $B_{z}$ variations in ACE (Figure 10) with velocity variations in ThE suggests that they penetrate from the interplanetary medium into the magnetosphere.

Penetration of Pc5 waves from the interplanetary medium into the magnetosphere has been discussed in [Kepko et al., 2002; Kepko, Spence, 2003; Kessel et al., 2004]. In [Kepko et al., 2002; Kepko, Spence, 2003], analysis of a series of geomagnetic pulsations caused by $P_{\mathrm{d}}$ variations has revealed that discrete frequencies of intramagnetospheric resonances of $0.7,1.3,1.9,2.6$, and $3.4 \mathrm{mHz}$ are observed in SW density variations and presumably reflect the existence of structures of certain dimensions in the interplanetary medium. Kepko, Spence [2003] have converted the variation frequencies to equivalent radial dimensions of corresponding structures in SW and obtained the following values: 150, 200, 300, and 500-600 Mm. This result has been confirmed by the statistical analysis of SW density data for 11 years carried out in [Viall et al., 2008]. The authors determined the most frequently observed statistically significant radial dimensions of SW structures: 73, 120, 136, and $180 \mathrm{Mm}$ for slow SW and 187, 270, and 400 $\mathrm{Mm}$ for fast SW. Periods with oscillations in our event (20-30 min) correspond to radial dimensions of irregularities (528-792 Mm) and are located on the edge (in the tail) of distribution of radial dimensions of the disturbances [Viall et al., 2008]. The main frequency of the oscillations in our event $(0.6-0.7 \mathrm{mHz})$ is close to the first harmonic of intramagnetospheric resonances.

Simultaneous measurements of the plasma density $n$ and the magnetic field modulus $B$ are typical of compression MHD waves: fast (FM) and SM. In the case of FM waves, these parameters vary in phase; whereas in the case of SM waves, in antiphase [Baumjohann, Treumann, 1996], as observed in our event in the interplanetary medium (Figure 2) and in the magnetosphere (Figure 7). Leonovich et al. [2015] state that only an FM wave with a small azimuthal wave number can penetrate into the magnetosphere without a significant decrease in amplitude. In the magnetosphere, it can transform into Alfvén and SM waves on resonant magnetic shells. However, the question about possible direct penetration of SM wave into the magnetosphere remains open. In our event unlike that analyzed in [Moiseev et al., 2016], we did not observe significant $P_{\mathrm{d}}$ variations, SW velocity did not exceed the mean value, which was obviously not enough for the development of instability at the boundary of the magnetosphere due to the SW flow around it. Perhaps the interplanetary medium conditions that give rise to generation of substorm activity due to reconnection favor the SM wave penetration into the magnetosphere.

The compression nature of the waves in the magnetosphere (Figure 9) and their scale are typical of irregularities from the source that has a small transverse wavelength, for example, from particle injections. Such oscillations cannot be recorded by ground-based magnetometers.

Thus, in this event oscillations of two types were simultaneously excited: SM waves observed in SW and in the magnetosphere, and oscillations of auroral electrojets (in the ionosphere) recorded on Earth - Hall currents excited by Alfvén oscillations of field-aligned currents. Yeoman, Wright [2000], using CUTLASS HFradar data, have identified three different waves in one event. The first, large-scale, was from the external source, whereas the second and third, small-scale, were from the internal sources. Beharrell et al. [2010], using scanning riometer data, have studied small-scale oscillations with azimuthal wave numbers up to 380 . The authors suggested that the observed small-scale oscillations were secondary, excited by primary oscillations in the magnetospheric cavity, which, in turn, were caused by $P_{\mathrm{d}}$ pulses. Compression disturbances of the magneto- 
spheric cavity transform into toroidal oscillations of the Alfvén mode; the latter direct their energy to resonant protons through the Landau damping mechanism. After primary oscillations damp out, protons through inverse Landau damping give their energy to small-scale secondary oscillations. The mechanism of generation of secondary oscillations in the event considered might be similar to the mechanism proposed by Beharrell et al. [2010], especially that the SM-wave velocity is close in magnitude to the thermal velocity of plasma ions, and therefore the wave can transfer its energy to them. In our event, the resonant proton energy determined from ThE data is $15.7 \mathrm{keV}$, and is in line with the maximum peak of resonant proton distribution obtained in [Beharrell et al., 2010].

\section{CONCLUSION}

From this analysis we can draw the following conclusions.

- Ground-based geomagnetic observations have shown azimuthal propagation of $\mathrm{Pi} 3$ pulsations to the east in the post-midnight sector and to the west in the premidnight sector with a velocity $0.6-10.6 \mathrm{~km} / \mathrm{s}$. Analysis of meridional propagation has established the fact of equatorward propagation with a velocity $0.75-7.87 \mathrm{~km} / \mathrm{s}$, both azimuthal and meridional velocities increasing with decreasing latitude. Such dynamics of the azimuthal and meridional propagation components suggests that plasma generally propagates in the magnetosphere to the equator from the midnight meridian.

- Compression mode magnetic field oscillations were recorded in Earth's magnetosphere on the dawn side in the range of periods of ground pulsations.

- We assume that oscillations in this event were caused by penetration of waves from SW due to reconnection and transformation in Earth's magnetosphere via wave - particle interaction.

We are grateful to Klimushkin D.Yu. and Leonovich A.S. for discussion of this article. We thank managers of the following projects for providing access to data: CANMOS, Geomagnetism Unit of the Geological Survey of Canada; GIMA; Intermagnet; USGS, Jeffrey J. Love; and $210 \mathrm{MM}$ (MAGDAS) data, and data set of the satellite observations from CDAWEB (DJ McComas, R. Lepping, K. Ogilvi, G. Paschmann, and G. Reeves). This work was partially funded by RFBR grants No. 18-45-140037 (MAV), No.18-42-140002 (SSA), and No. 18-05-00437 (MVV).

\section{REFERENCES}

Alimaganbetov M., Streltsov A.V. ULF waves observed during substorms in the solar wind and on the ground. $J$. Atmosph. Solar-Terr. Phys. 2018, vol. 181, pp. 10-18.

Angelopoulos V., Chapman J.A., Mozer F.S., Scudder J.D., Russell C.T., Tsuruda K., et al. Plasma sheet electromagnetic power generation and its dissipation along auroral field lines. $J$. Geophys. Res. 2002, vol. 107, p. 1181. DOI: 10.1029/ 2001JA900136.

Baumjohann W., Treumann R.A. Basic Space Plasma Physics. London: Imperial College Press, 1996. 340 p. DOI: $10.1142 / \mathrm{p} 015$.
Beharrell M., Kavanagh A.J., Honary F. On the origin of high-m magnetospheric waves. J. Geophys. Res.: Atmospheres. 2010, vol. 115. DOI: 10.1029/2009JA014709.

Belakhovsky V.B., Pilipenko V.A., Samsonov S.N. Pi3 pulsations and their correlation with fluxes of charged particles in the magnetosphere and ionosphere. Proc. XXXVIII Annual Seminar "Physics of Auroral Phenomena". Apatity. 2015, pp. 71-74. (In Russian).

Coronity F.V., Kennell C.F. Changes of magnetospheric configuration during the substorm growth phase. J. Geophys. Res. 1972, vol. 77, pp. 3361-3370.

Gjerloev J.W. The SuperMAG data processing technique. J. Geophys. Res. 2012, vol. 117, A09213, DOI: 10.1029/ 2012JA017683.

Guglielmi A.V., Zolotukhina N.A. Excitation of Alfvén oscillations in the magnetosphere by asymmetric ring current. Issledovaniya po geomagnetizmu, aeronomii i fizike Solntsa [Research on Geomagnetism, Aeronomy and Solar Physics]. 1980, iss. 50, pp. 129-138. (In Russian).

Hasegawa A. Drift mirror instability in the magnetosphere. Phys. Fluids. 1969, vol. 12. pp. 2642-2650.

Keiling A. Alfvén waves and their roles in the dynamics of the Earth's magnetotail: A review. Space Sci. Rev. 2009, vol. 142, pp. 73-156.

Keiling A., Parks G.K., Wygant J.R., Dombeck J., Mozer F.S., Russell C.T., Streltsov A.V., Lotko W. Some properties of Alven waves: Observations in the tail lobes and the plasma sheet boundary layer. J. Geophys. Res. 2005, vol. 110, A10S11. DOI: 10.1029/2004JA010907.

Kepko L., Spence H.E., Singer H.J. ULF waves in the solar wind as direct drivers of magnetospherc pulsations. Geophys. Res. Lett. 2002, vol. 29, p. 1197. DOI: 10.1029/ 2001 GL014405.

Kepko L., Spence H.E. Observations of discrete, global magnetospheric oscillations directly driven by solar wind density variations. J. Geophys. Res. 2003, vol. 108, p. 1257. DOI: 10.1029/2002JA009676.

Kessel R.L., Mann I.R., Fung S.F., Milling D.K., O'Connell N. Correlation of Pc5 wave power inside and outside the magnetosphere during high speed streams. Ann. Geophys. 2004, vol. 22, pp. 629-641.

Kiselev B.V., Raspopov O.M., Excitation of Pi3 pulsations during substoms. Proc. IAGA Meeting of Unmanned Observatories in Antarctica. Tokyo. 1976, p. 88.

Klibanova Yu.Yu., Mishin V.V., Tsegmed B. Peculiarities in daytime observed during solar wind pulse against the background of the August 1, 1998 substorm. Kosmicheskie issledovaniya [Cosmic Res.]. 2014, vol. 52, no. 6. pp. 459-467. (In Russian).

Kostarev D.V., Mager P.N. Drift-compression waves propagating in the direction of energetic electron drift in the magnetosphere. Solar-Terrestrial Physics. 2017, vol. 3, no. 3, pp. 18-27. DOI: 10.12737/stp-33201703.

Kozlovsky A., Lakkala T., Kangas J., Aikio A. Response of the quiet auroral arc motion to ionospheric convection variations. J. Geophys. Res. 2001. vol. 106, pp. 21463-21474.

Leonovich A.S., Mishin V.V., Cao J.B. Penetration of magnetosonic waves into the magnetosphere: influence of a transition layer. Annales Geophysicae. 2003, vol. 21, pp. 10831093.

Leonovich A.S., Mazur V.A., Kozlov D.A. MHD waves in the geomagnetic tail: A review. Solnechno-zemnaya fizika [Solar-Terrestrial Physics]. 2015, vol. 1, iss. 1, pp. 4-22. (In Russian).

Li W., Thorne R.M., Bortnik J., Nishimura Y., Angelopoulos V. Modulation of whistler mode chorus waves: 1. Role of compressional Pc4-5 pulsations. J. Geophys. Res. 2011, vol. 116, A06205. DOI: 10.1029/2010JA016312. 
Mager P.N., Klimushkin D.Yu. Generation of Alfvén waves by a moving plasma inhomogeneity in the magnetosphere. Fizika plazmy [Plasma Physics Rep.]. 2007. vol. 33, no. 5, pp. 435-442.

Mager P.N., Klimushkin D.Yu., Kostarev D.V. Drift-compressional modes generated by inverted plasma distributions in the magnetosphere. J. Geophys. Res. Space Phys., 2013, vol. 118, pp. 4915-4923. DOI: 10.1002/jgra.50471.

Makarov G.A., Solovyev S.I., Engebretson M., Yumoto K. Azimuth propagation of geomagnetic sudden pulse in high latitudes at the December 15, 1995 sharp decrease in a solar wind density. Geomagnetizm i aeronomiya [Geomagnetism and Aeronomy]. 2002, vol. 42, no. 1, pp. 42-50. (In Russian).

McKenzie J. F.: Hydromagnetic wave interaction with the magnetopause and the bow shock. Planetary and Space Sci. 1970, vol. 18, pp. 1-23.

Mishin V.V. Accelerated motions of the magnetopause as a trigger of the Kelvin_Helmholtz instability. J. Geophys. Res. 1993, vol. 98, no. A12, pp. 21365-21372.

Mishin V.V. On wave energy flux into the magnetosphere under the action of solar wind pressure pulsations. Issledovaniya po geomagnetizmu, aeronomii i fizike Solntsa [Research on Geomagnetism, Aeronomy and Solar Physics]. 1996. iss. 104. pp. 182-185. (In Russian).

Moiseev A.V., Baishev D.G., Mullayarov V.A., Samsonov S.N., Iozumi T., Ioshikawa A., et al. Development of compression long-period pulsations at the recovery phase of the May 23, 2007 magnetic storm. Kosmicheskie issledovaniya [Cosmic Res.]. 2016, vol. 54, no. 1, pp. 34-43. (In Russian).

Parkhomov V.A., Borodkova N.L., Eselevich V.G., Eselevich M.V., Dmitriev A.V., Chilikin V.E. Solar wind diamagnetic structures as a source of substorm-like disturbances. J. Atmos. Sol. Ter. Phys. 2018, vol. 181, pp. 55-67.

Parkhomov V.A., Mishin V.V., Leonovich A.S., Nikolaeva N.C., Solovyev S.I. Magnetospheric response in long-period geomagnetic pulsations observed during multiple crossing of the magnetopause by INTERBALL-1 satellite. Solnechno-zemnaya fizika [Solar-Terrestrial Physics]. 2005, iss. 8, pp. 161-163. (In Russian).

Pytte T., McPherron R.L., Hones E.W.Jr., West H.I.Jr. Multiple-satellite studies of magnetospheric substorms. III. Distinction between polar substorms and convection-driven negative bays. J. Geophys. Res. 1978, vol. 83, no. A2, pp. $663-$ 679.

Reeves G.D., Henderson M.G., McLachlan P.S., Belian R.D., Friedel R.H.W., Korth A. Radial propagation of substorm injections. Proc. the Third International Conference on Substorms. Eur. Space Agency Spec. Publ., 1996, ESA SP-389, pp. 579.

Rostoker G., Barichello J.C. Seasonal and diurnal variation of Ps6 magnetic disturbances. J. Geophys. Res. 1980, vol. 85, p. 161.

Russell A.J.B., Wright A.N., Streltsov A.V. Production of small-scale Alfvén waves by ionospheric depletion, nonlinear magnetosphere-ionosphere coupling and phase mixing. $J$. Geophys. Res. 2013, vol. 118, pp. 1450-1460. DOI: 10.1002/ jgra.50168.

Saito T. Long-period irregular magnetic pulsations, Pi3, Space Sci. Rev. 1978. p. 427.

Saito T., Matsushita S. Geomagnetic pulsations associated with sudden commencements and sudden impulses. Planet. Space Sci. 1967, vol. 15. pp. 573-587.

Sergeev V.A., Pellinen R.J., Pulkkinen T.I. Steady magnetospheric convection: A review of recent results. Space Sci. Rev. 1996, vol. 75, p. 551.

Solovyev S.I., Baishev D.G., Barkova E.S., Engebretson M.J., Posch J.L., Hughes W.J., Yumoto K., Pilipenko V.A.
Structure of disturbances in the dayside and nightside ionosphere during periods of negative interplanetary magnetic field B. J. Geophys. Res.: Space Phys. 1999. V. 104. P. 28019 28039. DOI: 10.1029/1999JA900286.

Spanswick E., Donovan E., Liu W., Wallis D., Aasnes A., Hiebert T., Jackel B., Henderson M., Frey H. Substorm Associated Spikes in High Energy Particle Precipitation The Inner Magnetosphere: Physics and Modeling. Geophysical Monograph Series, AGU, 2005, DOI: 10.1029/155GM24.

Van de Hulst H.C. Problems of Cosmical Aerodynamics. Dayton, OH: Central Air Documents Office, 1951. p. 45.

Viall N.M., Kepko L., Spence H.E. Inherent length-scales of periodic solar wind number density structures. J. Geophys. Res. 2008, vol. 113, no. A07101. DOI: 10.1029/2007JA012881.

Woch J., Kremser G., Pokhotelov O.A., Pilipenko V.A., Amata E. Curvature-driven drift mirror instability in the magnetosphere. Planet. Space Sci. 1988. vol. 36. pp. 383-393.

Woch J., Kremser G., Korth A. A comprehensive investigation of compressional ULF waves observed in the ring current. $J$. Geophys. Res. 1990. vol. 95, pp. 15113-15132. DOI: 10.1029/JA095iA09p15113.

Wygant J.R., Keiling A., Cattell C.A., Lysak R.L., Temerin M., Mozer F.S., et al. Evidence for kinetic Alfvén waves and parallel electron energization at 4-6 RE altitudes in the plasma sheet boundary layer. J. Geophys. Res. 2002, vol. 107, p. 1201. DOI: 10.1029/2001JA900113.

Yeoman T., Tian M., Lester M., Jones T.B. A study of Pc5 hydromagnetic waves with equatorward phase propagation. Planetary and Space Sci. 1992, vol. 40, no. 6. pp. 797-810.

Yeoman T.K., Wright D.M. ULF waves with drift resonance and drift-bounce resonance energy sources as observed in artificially-induced HF radar backscatter. Ann. Geophys. 2000, vol. 19, pp.159-170. 2020).

URL: http://supermag.jhuapl.edu/mag (accessed March 12

URL: http://www.serc.kyushu-u.ac.jp/magdas (accessed March 12 2020).

URL: http://themis.ssl.berkeley.edu/gmag_desc.shtml (accessed March 12 2020).

URL: http://cdaweb.gsfc.nasa.gov (accessed March 12 2020).

URL: https://www.ngdc.noaa.gov/stp/satellite/goes/dataaccess.html (accessed March 12 2020).

URL: https://www.mathworks.com/help/signal/ref/filtfilt. html (accessed March 12 2020).

How to cite this article

Moiseev A.V., Starodubtsev S.A., Mishin V.V. Features of excitation and azimuthal and meridional propagation of long-period pi3 oscillations of the geomagnetic field on December 8, 2017. SolarTerrestrial Physics. 2020. Vol. 6. Iss. 3. P. 46-59. DOI: 10.12737/stp63202007. 